\title{
Ten years of Swift: a universal scaling for short and long gamma-ray bursts $\left(E_{X, \text { iso }}-E_{\gamma, \text { iso }}-E_{p k}\right)$
}

\author{
Elena Zaninoni* \\ ICRANet-Rio, Centro Brasileiro de Pesquisas Fisicas, Rua Dr. Xavier Sigaud 150, 22290-180 \\ Rio de Janeiro, Brazil \\ E-mail: elena.zaninoni@gmail.com
}

Maria Grazia Bernardini

INAF, Osservatorio Astronomico di Brera, via Bianchi 46, 23807 Merate, LC, Italy

E-mail: grazia.bernardini@brera.inaf.it

\section{Raffaella Margutti}

Harvard-Smithsonian Center for Astrophysics, 60 Garden Street, Cambridge, MA 02138, USA

E-mail: rmargutti@cfa.harvard.edu

\section{Lorenzo Amati}

INAF, Istituto di Astrofisica Spaziale e Fisica Cosmica, Bologna, Via Gobetti 101, I-40129

Bologna, Italy

E-mail: amati@iasfbo.inaf.it

From the comprehensive statistical analysis of Swift X-ray light-curves collected from the launch of the Swift satellite until the end of 2010, we found a three parameter correlation between the isotropic energy emitted in the rest frame $1-10^{4} \mathrm{keV}$ energy band during the prompt emission $\left(\mathrm{E}_{\gamma, \text { iso }}\right)$, the rest frame peak of the prompt emission energy spectrum $\left(\mathrm{E}_{\mathrm{pk}}\right)$, and the X-ray energy emitted in the rest frame $0.3-30 \mathrm{keV}$ observed energy band $\left(\mathrm{E}_{\mathrm{X}, \text { iso }}\right)$. The importance of this scaling law is that it is followed by both long and short GRBs, and, at the same time, involves prompt and afterglow emission quantities. Therefore there are some properties which are shared by long and short GRBs as a whole. We updated this correlation considering all GRBs observed until June 2014, confirming the existence of this scaling law, and examining some particular GRBs, as 090426 and 100816A. We also discuss the physics that is driving this correlation.

Swift: 10 Years of Discovery,

2-5 December 2014

La Sapienza University, Rome, Italy

\footnotetext{
* Speaker.
} 


\section{Introduction}

The Swift satellite [1], launched on November 2004, opened a new era for the study and understanding of gamma-ray busts (GRBs), detecting more than 900 GRBs until now. Thanks to its data, many correlations involving prompt and afterglow emission quantities were investigated (e.g. $[2,3,4,5,6])$.

From the comprehensive statistical analysis of Swift X-ray light-curves collected from December 2004 until December 2010 ([7], hereafter M13), we found a three-parameter correlation between the isotropic energy emitted in the rest frame $1-10^{4} \mathrm{keV}$ energy band during the prompt emission $\left(\mathrm{E}_{\gamma, \text { iso }}\right)$, the rest frame peak of the prompt emission energy spectrum $\left(\mathrm{E}_{\mathrm{pk}}\right)$, and the $\mathrm{X}$-ray energy emitted in the rest frame $0.3-30 \mathrm{keV}$ observed energy band $\left(\mathrm{E}_{\mathrm{X}, \text { iso }}\right)$. The uniqueness of this correlation is that accommodates long, short, and low-energetic GRBs, and, at the same time, involves prompt and afterglow emission quantities, suggesting that there are some properties which are shared by the GRB class as a whole ([8], hereafter B12, M13).

We considered all GRBs observed until June 2014 as in M13 and we selected only GRBs that have: i) secure redshift measurement; ii) measured $\mathrm{E}_{\mathrm{pk}}$; iii) complete $\mathrm{X}$-ray light-curve ${ }^{1}$. In this way, we obtained a new sample composed of 81 long GRBs, 12 short GRBs and 2 GRBs with uncertain classification ${ }^{2}$. The new sample contains $\sim 35 \%$ more GRBs than the previous one; in particular the sample of short GRBs doubled (Table 1).

Uncertainties are given at 68 per cent confidence level (c.l.) unless explicitly mentioned. Standard cosmological quantities have been adopted: $\mathrm{H}_{0}=70 \mathrm{~km} \mathrm{~s}^{-1} \mathrm{Mpc}^{-1}, \Omega_{\Lambda}=0.7$ and $\Omega_{\mathrm{M}}=$ 0.3 .

\section{The three-parameter correlation}

The three-parameter correlation (Figure 1) involves $\mathrm{E}_{\mathrm{X} \text {,iso }}, \mathrm{E}_{\gamma \text {,iso }}$, and $\mathrm{E}_{\mathrm{pk}} . \mathrm{E}_{\mathrm{pk}}$ and $\mathrm{E}_{\gamma, \text { iso }}$ are calculated as described in [3]. For short GRBs 080123, 090423, 100625A, 111117A, and 130603B, we consider the values of $\mathrm{E}_{\mathrm{pk}}$ and $\mathrm{E}_{\gamma, \text { iso }}$ reported in [9]. $\mathrm{E}_{\mathrm{X} \text {,iso }}$ is the X-ray energy emitted in the 0.3-30 keV band in the rest frame and we calculated it as in M13 (for more details see also [10, 11]).

The correlation is derived using the method of D'Agostini [12], which considers an intrinsic scatter $\sigma_{\text {ext }}$ that accounts for the possible contribution of hidden variables. We obtained:

$$
\begin{aligned}
\log \left[\frac{E_{X, \text { iso }}}{\text { erg }}\right]= & (0.97 \pm 0.06) \log \left[\frac{E_{\gamma, \text { iso }}}{\text { erg }}\right] \\
& -(0.57 \pm 0.13) \log \left[\frac{E_{\mathrm{pk}}}{\mathrm{keV}}\right]-(0.62 \pm 0.08)
\end{aligned}
$$

with an extra-scatter $\sigma_{\mathrm{ext}}=0.32 \pm 0.04$. Figure 1 shows a two-dimension representation of this relation respect to observations. As explained in previous papers (B12, M13), this correlation is

\footnotetext{
${ }^{1}$ Promptly re-pointed by Swift/XRT ( $\mathrm{t}_{\mathrm{rep}}<300 \mathrm{~s}$ ) and for which observations were not limited by any observing constraint (M13).

${ }^{2}$ GRBs with uncertain classification are GRB 090426 and GRB 100816A. A detailed discussion can be found in Sec. 3 .
} 
Ten years of Swift: a universal scaling for short and long gamma-ray bursts $\left(E_{\mathrm{X}, \mathrm{iso}}-E_{\gamma, \mathrm{iso}}-E_{\mathrm{pk}}\right)$ Elena Zaninoni

Table 1: List of 34 GRBs added to the old sample. Short GRBs are marked in boldface, while GRB with uncertain clssification is underlined.

GRB name

080123, 090426, 100117A, 100625A, 110106B, 110205A, 110213A, 110503A, 110715A, 110731A, 110801A, 110818A, 111107A, 111117A, 111209A, 111228A, 120119A, 120326A, 120804A, 120712A, 120802A, 120811C, 121128A, 130408A, 130427A, 130505A, 130603B, 130701A, 130831A, 130907A, 130925A, 131030A, 140206A, 140419A

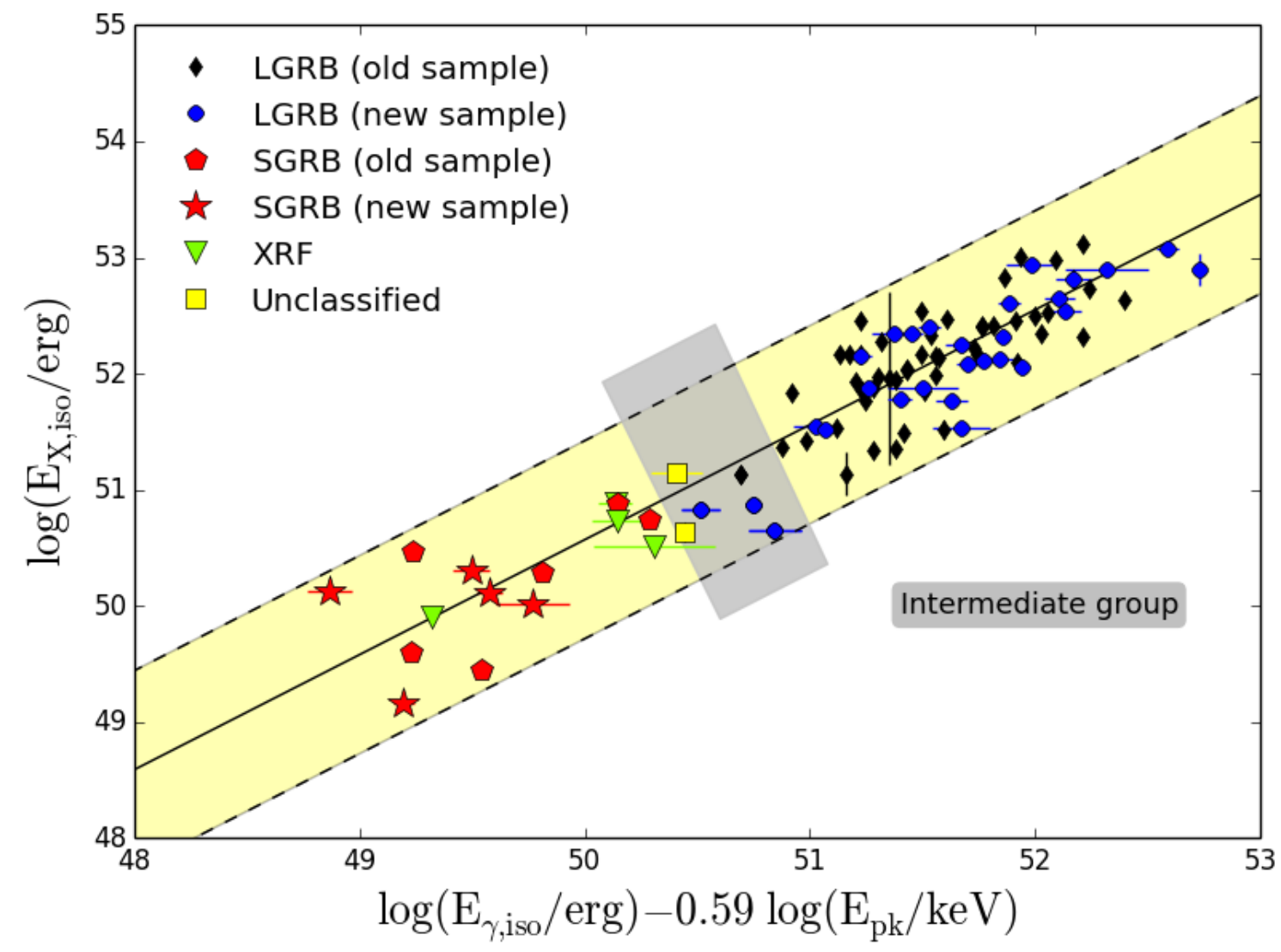

Figure 1: Three-parameter correlation for the sample of 81 long GRBs (black diamonds for the old sample, blue dots for the new sample, and green triangles for low-energetic GRBs (i.e. X-ray flashes (XRFs), GRBs with $\mathrm{E}_{\gamma \text {,iso }} \lesssim 10^{52} \mathrm{erg}$ ), 12 short GRBs (red pentagons for the old sample and red stars for the new sample), and two GRBs with uncertain classification (yellow squares). The black solid line is the best-fitting function $\mathrm{y}=0.99(\mathrm{x}-0.59 \mathrm{z})-0.63$ and the yellow area marks the $2 \sigma$ region. The gray area indicates the intermediate group.

robust, spanning four orders of magnitude in $\mathrm{E}_{\mathrm{X} \text {,iso }}$ and $\mathrm{E}_{\mathrm{pk}}$, and six orders of magnitude in $\mathrm{E}_{\gamma, \text { iso }}$, and combines both short and long GRBs in a common scaling.

\section{The intermediate group}

In the previous version of the three-parameter correlation, there was a gap between short and long GRBs. Due to the updated sample, now this area is occupied by three new long GRBs 

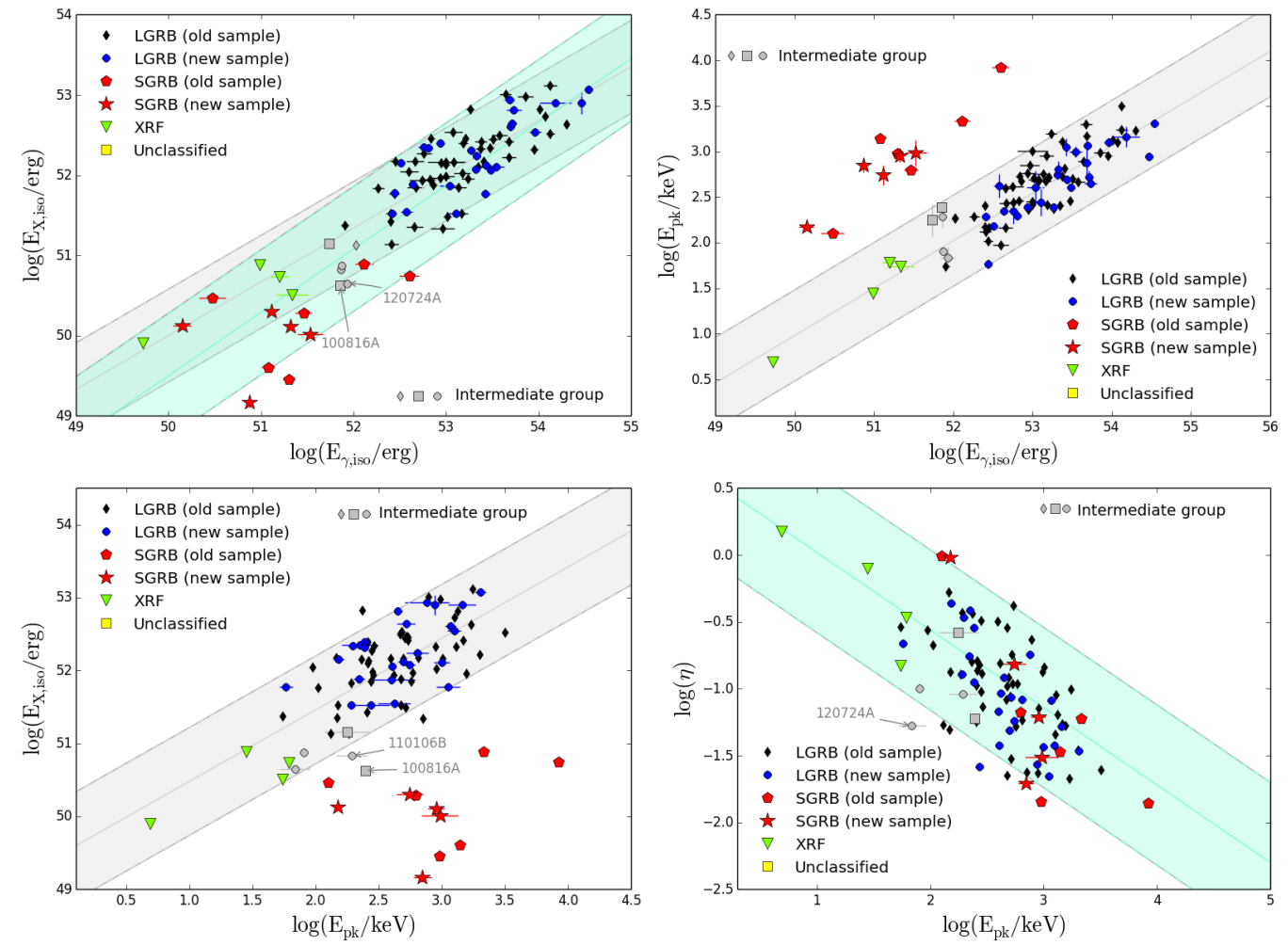

Figure 2: Lightblue and gray solid lines are the best-fitting functions, while gray and lightblue areas mark the $2 \sigma$ region for the best fit function obtained for the sample of long GRBs and the complete sample, respectively. The name of the outlier GRBs is indicated. Top left, $\mathrm{E}_{\gamma, \text { iso }}-\mathrm{E}_{\mathrm{X} \text {,iso }}$ relation: $\log \mathrm{Y}=0.82 \mathrm{Log}$ $\mathrm{X}-0.85(\sigma=0.39)$ (all GRBs); $\log \mathrm{Y}=0.67 \log \mathrm{X}-0.66(\sigma=0.35)$ (long GRBs). Top right, $\mathrm{E}_{\gamma, \text { iso }}-\mathrm{E}_{\mathrm{pk}}$ relation: $\log \mathrm{Y}=0.52 \log \mathrm{X}+0.02(\sigma=0.18)$ (long GRBs). Bottom left, $\mathrm{E}_{\mathrm{pk}}-\mathrm{E}_{\mathrm{X}, \text { iso }}$ relation: $\log \mathrm{Y}=1.00$ $\log \mathrm{X}-0.68(\sigma=0.34)$ (long GRBs). Bottom right, $\mathrm{E}_{\mathrm{pk}}-\varepsilon$ relation: $\log \mathrm{Y}=0.62 \log \mathrm{X}-0.65(\sigma=0.35)$ (all GRBs).

(110806B, 120724A, 130831A), two GRBs with uncertain classification (090426, 100816A), and a long GRB belonging to the old sample (080916) (Figure 1, gray area).

In particular, analysing the two-parameter correlation between $\mathrm{E}_{\mathrm{X} \text {,iso }}, \mathrm{E}_{\gamma, \text { iso }}$ and $\mathrm{E}_{\mathrm{pk}}$ (Figure 2), respectively, we notice that:

1. GRBs 090426, 080916, and 130831A behave as long GRBs. Indeed, 080916 and 130831A are characterized by a $\mathrm{T}_{90}>2 \mathrm{~s}$ and a soft spectrum; moreover, a supernova was detected in association with GRB 130831A [13]. These two GRBs have also a redshift $<1$. The classification of GRB 090426 is debated (e.g., [14, 15, 16, 17, 18, 9]).

2. The other three GRBs show a lower X-ray energy respect that is expected for long GRBs having their $\mathrm{E}_{\mathrm{pk}}$ or $\mathrm{E}_{\gamma, \text { iso }}$, respectively.

For a GRB event, the efficiency of the process is defined as the ratio between the prompt emission energy and the outflow kinetic energy (e.g., [19]). Since the kinetic energy could be computed considering the afterglow emission, we define the inverse of the efficiency as $\varepsilon=\mathrm{E}_{\gamma}$,iso 
/ $\mathrm{E}_{\mathrm{X}, \text { iso }}$ and we plot this quantity against the peak energy $\mathrm{E}_{\mathrm{pk}}$ (Figure 2, bottom right). As we showed in our previous works (B12, M13), we have two new groups: one of low-energetic GRBs which are less efficient and occupy the top left part of the plane, and the other group composed of short and long GRBs, which have similar efficiencies. From this plot we can see that GRBs of the intermediate group share the area with efficient bursts.

\section{Physics and models}

The photospheric model considers how the GRB spectrum in the optically thick phase can be modified by the interaction of the radiation field with the leptonic component of the outflow, before it is released at the photosphere [20, 21, 22, 23]. The simulations made by Lazzati et al. [24] can reproduce the three-parameter correlation since the radiative efficiency of brighter bursts is higher than that of weaker bursts. However, for adequately comparing the observations and the simulations, it is necessary to assume a value for the electron equipartition parameter $\varepsilon$ [24]. They show that by adopting the fiducial value $\varepsilon=0.1$, a good agreement between the simulation results and the observed values is obtained.

In the Cannonball (CB) model [25, 26, 27], the Inverse Compton scattering caused by the interaction between electrons of the CB plasma and the light in the near ambient of the supernova is responsible of the gamma-ray prompt emission of GRBs, while the afterglow emission is related to the synchrotron radiation of electrons swept-in and accelerated in the CBs. In this model, the three-parameter correlation is simply the combination of the two parameter correlations of kinetic origin that are followed by both long and short GRBs, even if with different normalizations, and so it depends on the large Doppler boosting and the relativistic beaming that strongly influenced the observed radiation [28].

\section{Summary and conclusions}

In this work we updated the three-parameter correlation (B12, M13) considering GRBs detected until June 2014: the new sample contains about 35\% more GRBs than the original one, and, in particular, the number of short GRBs doubles (81 long GRBs, 12 short GRBs, and 2 GRBs with uncertain classification). Due to the increment of the number of bursts in our sample, the area between long and short GRBs is filled by three new long GRBs, two GRBs with uncertain classification, and a GRB belonging to the old sample. For a better understanding of the properties of these six intermediate GRBs, we considered also the two parameter correlations involving $\mathrm{E}_{\mathrm{X} \text {,iso }}$, $\mathrm{E}_{\gamma, \text { iso }}$ and $\mathrm{E}_{\mathrm{pk}}$. From this analysis, for instance, we found out that GRB 090426 lies in the group of long GRBs, despite the short duration of its gamma-ray emission, and GRB 100816A shows a low X-ray energy respect that of long GRBs. Moreover, GRBs of the intermediate group are efficient as long and short GRBs. The three-parameter correlation can be a useful tool for classifying GRBs and understanding their properties as a group. Finally, we briefly report the role of the three parameter correlation in the photospheric model [24] and in the Cannonball model [28]. A detailed description and analysis of the three-parameter correlation and its implications will be presented in Zaninoni et al. (in preparation). 


\section{Acknowledgments}

This work made use of data supplied by the UK Swift Science Data Centre at the University of Leicester. EZ acknowledges the support by the International Cooperation Program CAPESICRANet financed by CAPES - Brazilian Federal Agency for Support and Evaluation of Graduate Education within the Ministry of Education of Brazil and by ICRANet that allows her to partecipate to this meeting.

\section{References}

[1] N. Gehrels, G. Chincarini, P. Giommi, et al., The Swift Gamma-Ray Burst Mission, ApJ 611 (2004) 1005.

[2] D. E. Reichart, D. Q. Lamb, E. E. Fenimore, et al., A Possible Cepheid-like Luminosity Estimator for the Long Gamma-Ray Bursts, ApJ 552 (2001) 57 [arXiv:astro-ph/ 0004302 ].

[3] L. Amati, F. Frontera, M. Tavani, et al., Intrinsic spectra and energetics of BeppoSAX Gamma-Ray Bursts with known redshifts, A\&A 390 (2002) 81 [arXiv: astro-ph/0205230].

[4] G. Ghirlanda, G. Ghisellini \& D. Lazzati, The Collimation-corrected Gamma-Ray Burst Energies Correlate with the Peak Energy of Their $v F_{v}$ Spectrum, ApJ 616 (2004) 331 [arXiv:astro-ph/0405602].

[5] D. Yonetoku, T. Murakami, T. Nakamura, et al., Gamma-Ray Burst Formation Rate Inferred from the Spectral Peak Energy-Peak Luminosity Relation, ApJ 609 (2004) 935 [arXiv:astro-ph/0309217].

[6] M. G. Dainotti, V. F. Cardone, \& S. Capozziello, A time-luminosity correlation for $\gamma$-ray bursts in the $X$-rays, MNRAS 391 (2008) L79 [arXiv: 0809 . 1389].

[7] R. Margutti, E. Zaninoni, M. G. Bernardini, et al., The prompt-afterglow connection in gamma-ray bursts: a comprehensive statistical analysis of Swift X-ray light curves, MNRAS 428 (2013) 729 [arXiv: 1203.1059].

[8] M. G. Bernardini, R. Margutti, E. Zaninoni, \& G. Chincarini, A universal scaling for short and long gamma-ray bursts: $E_{X ; \text { iso }}-E_{\gamma ; i s o}-E_{p k}$, MNRAS 425 (2012) 1199 [arXiv: 1203.1060 ].

[9] P. D’Avanzo, R. Salvaterra, M. G. Bernardini, et al., A complete sample of bright Swift short gamma-ray bursts, MNRAS 442 (2014) 2342 [arXiv : 1405 . 5131].

[10] R. Margutti, M. G. Bernardini, R. Barniol Duran et al., On the average gamma-ray burst X-ray flaring activity, MNRAS 410 (2011) 1064 [arXiv: 1009.0172 ].

[11] E. Zaninoni (2013), Gamma-ray bursts and their X-ray and optical afterglow, Universitá degli Studi di Padova (2013) [http: / / paduaresearch. cab.unipd.it/5626/].

[12] G. D'Agostini, Fits, and especially linear fits, with errors on both axes, extra variance of the data points and other complications, ArXiv Physics e-prints (2005) [arXiv: phys ics / 0511182].

[13] S. Klose, A. Nicuesa Guelbenzu, T. Kruehler, et al., GRB Coordinates Network, 15320 (2013) 1.

[14] L. A. Antonelli, P. D’Avanzo, R. Perna, et al., GRB 090426: the farthest short gamma-ray burst?, $A \& A \mathbf{5 0 7}$ (2009) L45 [arXiv: 0911.0046$].$

[15] E. M. Levesque, J. S. Bloom, N. R. Butler, et al., GRB090426: the environment of a rest-frame 0.35-s gamma-ray burst at a redshift of 2.609, MNRAS 401 (2010) 963 [arXiv: 0907 . 1661]. 
[16] C. C. Thöne, S. Campana, D. Lazzati, et al., Variable Ly $\alpha$ sheds light on the environment surrounding GRB 090426, MNRAS 414 (2011) 479 [arXiv: 1101 . 3488].

[17] A. Nicuesa Guelbenzu, S. Klose, A. Rossi, et al., GRB 090426: Discovery of a jet break in a short burst afterglow, A\&A 531 (2011) LL6 [arXiv:1105.1312].

[18] D. Grupe, J. A. Nousek, P. Veres, B.-B. Zhang, \& N. Gehrels, Evidence for New Relations between Gamma-Ray Burst Prompt and X-Ray Afterglow Emission from 9 Years of Swift, ApJS 209 (2013) 20 [arXiv:1305.3236].

[19] N. M. Lloyd-Ronning \& B. Zhang, On the Kinetic Energy and Radiative Efficiency of Gamma-Ray Bursts, ApJ 613 (2004) 477 [astro-ph / 0404107 ].

[20] P. Mészáros, M. J. \& Rees, Steep Slopes and Preferred Breaks in Gamma-Ray Burst Spectra: The Role of Photospheres and Comptonization, ApJ 530 (2000) 292 [astro-ph/9908126].

[21] M. J. Rees, \& P. Mészáros, Dissipative Photosphere Models of Gamma-Ray Bursts and X-Ray Flashes, ApJ 628 (2005) 847 [astro-ph / 0412702 ].

[22] D. Giannios, Prompt emission spectra from the photosphere of a GRB, A\&A 457 (2006) 763 [astro-ph/0602397].

[23] D. Lazzati, B. J. Morsony \& M. C. Begelman, Very High Efficiency Photospheric Emission in Long-Duration $\gamma$-Ray Bursts, ApJ 700 (2009) L47 [arXiv : 0904 . 2779].

[24] D. Lazzati, B. J. Morsony, R. Margutti \& M. C. Begelman, Photospheric Emission as the Dominant Radiation Mechanism in Long-duration Gamma-Ray Bursts, ApJ 765 (2013) 103 [arXiv:1301.3920].

[25] A. Dar, \& A. de Rújula, Towards a complete theory of gamma-ray bursts, Physics Reports 405 (2004) 203 [astro-ph/0308248].

[26] S. Dado, A. Dar, \& A. De Rújula, Short Hard Gamma-Ray Bursts and their Afterglows ApJ 693 (2009) 311 [arXiv: 0807.1962$]$.

[27] S. Dado, A. Dar, \& A. De Rújula, The Diverse Broadband Light Curves of Swift Gamma-Ray Bursts Reproduced with the Cannonball Model, ApJ 696(2009) 994 [arXiv: 0809 . 4776].

[28] S. Dado, \& A. Dar, On the Recently Discovered Correlations between Gamma-Ray and X-Ray Properties of Gamma-Ray Bursts, ApJ 775 (2013) 16 [arXiv : 1203.5886 ]. 